\title{
Elevated Plasma Factor VIII in Non-Pyogenic Cerebral Venous Thrombosis after Head Trauma without Skull Fracture
}

\begin{abstract}
KipyoungJ eon', J ean Hee Kim', Kijeong Lee ${ }^{2}$, Kyu-Nam Park ${ }^{3}$, Bum-Soo Kim ${ }^{4}$, Yong Sam Shin ${ }^{5}$ and J aseong $\mathrm{Koo}^{\mathrm{l} *}$

'Department of Neurology, Seoul St. Mary's Hospital, The Catholic University of Korea, Seoul, Korea

${ }^{2}$ Department of Neurology, Eunpyeong St. Mary's

Hospital, The Catholic University of Korea, Seoul, Korea ${ }^{3}$ Department of Emergency Medicine, Seoul St. Mary's Hospital, The Catholic University of Korea, Seoul, Korea ${ }^{4}$ Department of Radiology, Seoul St. Mary's Hospital, The Catholic University of Korea, Seoul, Korea

${ }^{5}$ Department of Neurosurgery, Seoul St. Mary's Hospital, The Catholic University of Korea, Seoul, Korea

*Corresponding author: J aseong Koo, Department of Neurology, Seoul St. Mary's Hospital, The Catholic University of Korea, Seoul, Korea
\end{abstract}

Received: August 11, 2019; Accepted: September 18, 2019; Published: September 25, 2019

\begin{abstract}
Cerebral Venous Thrombosis (CVT) is a relatively uncommon but important cause of stroke that tends to affect young adults, especially women. Head trauma with or without skull fracture was reported to be triggering factors for CVT, but the underlying pathophysiology was not well elucidated. Endothelial injury and coexistent hypercoagulability were supposed to contribute to CVT after head trauma without skull fracture. We report a 49-year-old female patient who presented with headache with vomiting after head trauma and was initially diagnosed as post-traumatic Intracerebral Hemorrhage $(\mathrm{ICH})$, but subsequently, progressed to CVT that resulted in cerebral venous infarction with hemorrhagic transformation. Magnetic Resonance brain Venography (MRV) confirmed CVT in superior sagittal sinus as well as right transverse and sigmoid sinuses. She was treated with endovascular mechanical thrombectomy followed by anticoagulation. The coagulopathy panel was checked both in hospital and in outpatient clinic for evaluating the etiology underlying post-traumatic nonpyogenic CVT. Persistently elevated level of plasma Factor VIII was identified. We should consider that patients with recent head trauma history without skull fracture and coexistent hypercoagulability could develop CVT resulting in cerebral venous infarction with hemorrhagic transformation even when the patient showed no definite focal neurologic deficit or the patient's initial CT scan revealed no intracranial hemorrhage.
\end{abstract}

Keywords: Head Trauma; Cerebral Venous Thrombosis; Cerebral Venous Infarction; Hypercoagulability; Factor VIII

\section{Introduction}

Cerebral Venous Thrombosis (CVT) is a relatively uncommon but important cause of stroke that tends to affect young adults, especially women. The hypercoagulable state explains the increased tendency of developing cerebral venous thrombosis in various medical conditions such as thrombophilia, pregnancy, oral contraceptive usage, malignancy and rheumatologic diseases. Head trauma with or without skull fracture was also reported to be a triggering factor for CVT [1-3], but the underlying pathophysiology was not well elucidated. Endothelial injury and coexistent hypercoagulability possibly contributed to CVT after head trauma without skull fracture $[1,2]$. A few articles showed that CVT after head trauma could be complicated with cerebral venous infarction or delayed Intracerebral Hemorrhage (ICH) [3,4].

We report the case of a young female patient who presented with headache with vomiting after head trauma without skull fracture and was initially diagnosed as post-traumatic ICH, but subsequently progressed to CVT that resulted in acute venous infarction with hemorrhagic transformation. Persistently elevated plasma level of Factor VIII was identified and considered to be an underlying hypercoagulable condition that was responsible for non-pyogenic CVT after head trauma without skull fracture.

\section{Case Presentation}

A 49-year-old female patient visited our Emergency Room (ER) with a complaint of headache with vomiting after she had fallen from the bed during sleep and suffered head trauma. She had no remarkable findings in past medical history. She was discharged from ER and referred to outpatient clinic. She had no focal neurologic deficit on neurological examination, but brain $\mathrm{CT}$ was taken and revealed intracerebral hemorrhage in both frontal lobes (Figure 1A). CT brain Angiography (CTA) showed no abnormal findings in both head and neck arteries (Figure 1B). Enhanced T1-weighted brain MRI detected no definite thrombosis in cerebral venous sinuses including superior sagittal sinus (Figure $1 \mathrm{C}$ ). She was diagnosed as post-traumatic ICH and recovered after she was treated in a conservative manner.

After discharge, she visited ER again due to headache with nausea. CTA was taken immediately, but it only showed old cerebral lesions in both frontal lobes without acute hemorrhage. She was followed up in outpatient clinic three days later and expressed her symptom relief without focal neurological deficit. Five days later, she revisited outpatient clinic due to left hemiparesis beginning one day ago. T2-weighted brain MRI showed cerebral venous infarction with hemorrhagic transformation in right temporal lobe (Figure 2A). 3D Multi-Planar Reformatted (MPR) images visualized CVT in right transverse sinus and in superior sagittal and right sigmoid sinuses 

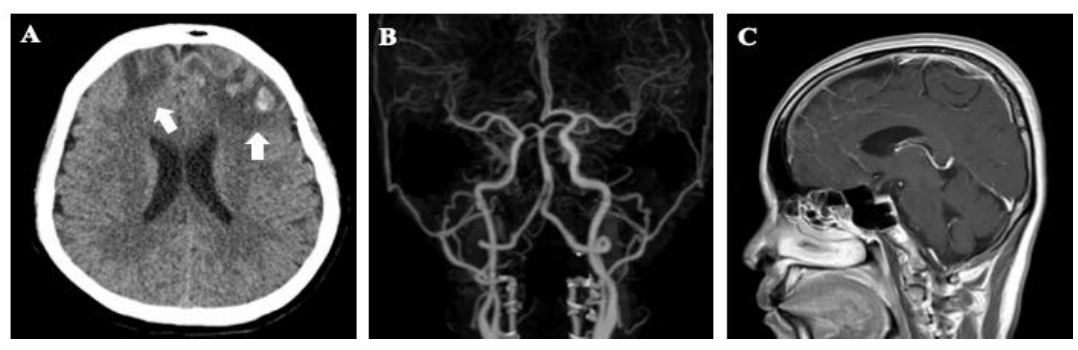

Figure 1: (A) Post-traumatic Intracerebral Hemorrhages (ICH) in both frontal lobes. (B) CT brain Angiography (CTA) showing no abnormal findings in head and neck arteries. (C) Enhanced T1-weighted brain MRI confirming no definite Cerebral Venous Thrombosis (CVT) in superior sagittal sinus.
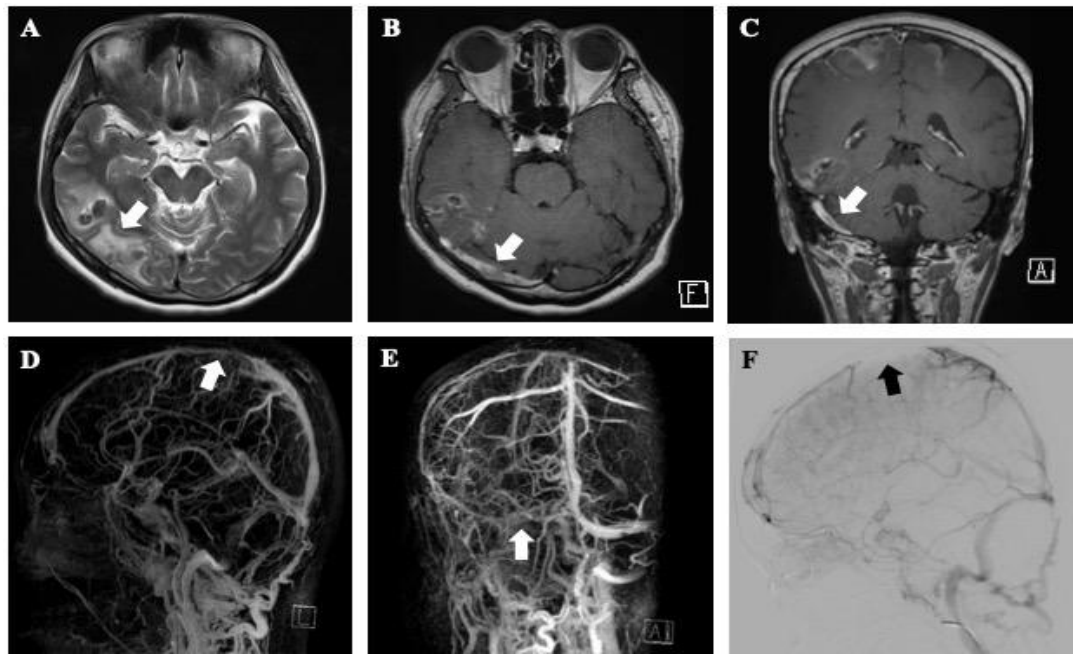

Figure 2: (A) T2-weighted brain MRI showing cerebral venous infarction with hemorrhagic transformation in right temporal lobe. 3D MPR images visualizing CVT (B) in right transverse sinus as well as (C) in superior sagittal and right sigmoid sinuses. MRV showing CVT (D) in superior sagittal sinus from the sagittal view as well as $(E)$ in right transverse and sigmoid sinuses from the anterior view. (F) DSA confirming CVT in superior sagittal sinus.

(Figures 2B,C). Magnetic Resonance brain Venography (MRV) confirmed CVT in superior sagittal sinus as well as right transverse and sigmoid sinuses (Figures 2D,E). Digital Subtraction Angiography (DSA) elucidated CVT in superior sagittal sinus again (Figure 2F).

We treated her immediately with endovascular mechanical thrombectomy through superior sagittal sinus followed by anticoagulation with intravenous Unfractionated Heparin (UFH) and oral warfarin (Figures 3A,B). The coagulopathy panel including protein $\mathrm{C}$, protein $\mathrm{S}$ and von-Willebrand's Factor (vWF) showed elevated plasma levels of D-dimer, Fibrinogen Degradation Product (FDP) and Factor VIII activity (>150\%; reference interval 60 150\%) as well as increased Prothrombin Time (PT) and activated Partial Thromboplastin Time (aPTT). Follow-up MRV was performed one week later and showed partial recanalization of CVT in superior sagittal sinus as well as right transverse and sigmoid sinuses (Figures $3 \mathrm{C}, \mathrm{D})$. She was discharged on the next day and advised to maintain oral warfarin daily with target INR 2.0-3.0. Two weeks after discharge, follow-up MRV confirmed that not only superior sagittal sinus, but also right transverse and sigmoid sinuses were fully recanalized under maintenance of oral warfarin (Figures 3E,F). The patient was regularly followed up in outpatient clinic and treated with aspirin after cessation of warfarin about six months later.

About one year later, we reevaluated the etiology underlying non-pyogenic CVT that was triggered by head trauma and resulted in cerebral venous infarction with hemorrhagic transformation. The coagulopathy panel was checked again and revealed the persistently elevated plasma level of Factor VIII activity whereas plasma levels of other factors in the coagulopathy panel were normalized. She has been regularly followed up in outpatient clinic with continued medication of aspirin.

\section{Discussion}

This case showed that non-pyogenic CVT could be triggered by head trauma without skull fracture and cause cerebral venous infarction with hemorrhagic transformation. Post-traumatic CVT could result either directly from head trauma or indirectly from post-traumatic ICH, which was possibly caused either by cerebral contusion or by hemorrhagic progression due to coagulopathy related to Traumatic Brain Injury (TBI) [5]. In this case, the coagulopathy panel was checked when the patient was diagnosed as cerebral venous infarction with hemorrhagic transformation. The results showed elevated plasma levels of D-dimer, FDP and Factor VIII activity as well as increased PT and aPTT. Follow-up coagulopathy panel was checked about one year later and showed persistently elevated plasma Factor VIII activity whereas the plasma levels of other factors in the coagulopathy panel were normalized. We considered that both endothelial injury caused by cerebral contusion and coagulopathy 

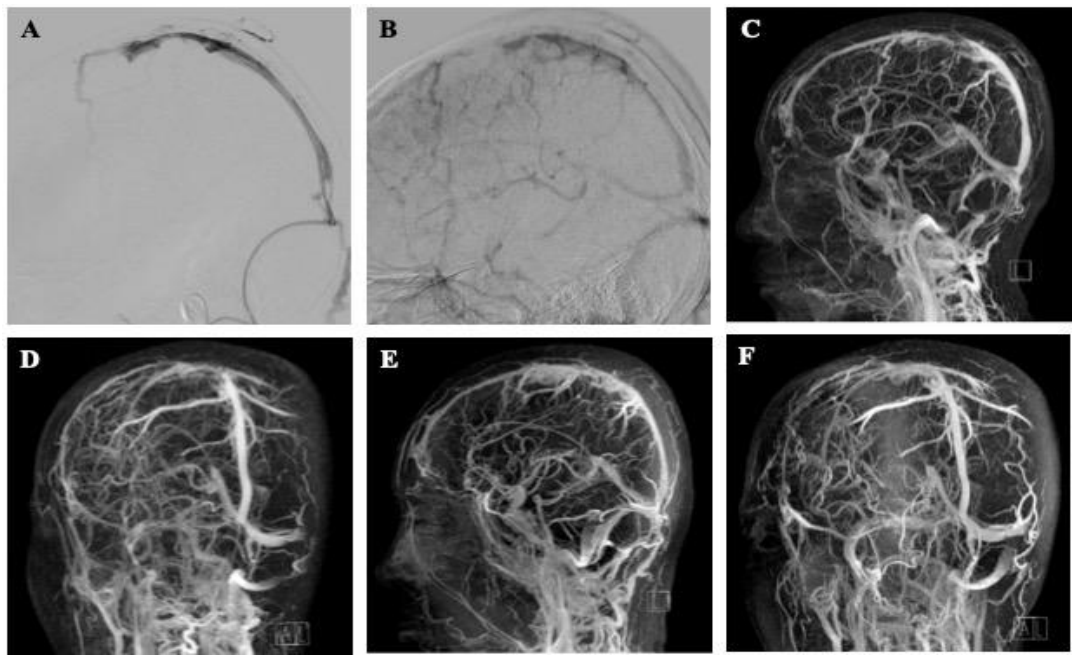

Figure 3: (A) Endovascular mechanical thrombectomy through superior sagittal sinus. (B) Partial recanalization of CVT in superior sagittal sinus after mechanical thrombectomy. Follow-up MRV before discharge showing partial recanalization of (C) superior sagittal sinus as well as (D) right transverse and sigmoid sinuses after intravenous Unfractionated Heparin (UFH) and oral warfarin treatment. Follow-up MRV two weeks after discharge showing full recanalization of (E) superior sagittal sinus as well as (F) right transverse and sigmoid sinuses under maintenance of oral warfarin treatment.

related to TBI might trigger post-traumatic CVT, but persistently elevated plasma Factor VIII in this case would be an important coexistent hypercoagulable condition underlying post-traumatic CVT that resulted in cerebral venous infarction with hemorrhagic transformation.

A variety of risk factors for CVT were documented in the literatures [6]. Head trauma with or without skull fracture was known to be an independent risk factor for CVT [1-3]. Head trauma with skull fracture could cause penetrating injury or compression of dural sinuses, which subsequently progressed to intramural hemorrhages or endothelial injuries that can predispose cerebral venous sinuses to thrombosis [7]. Head trauma without skull fracture was also reported to cause CVT with coexistent hypercoagulable states, intramural hemorrhages caused by rupture of small sinusoids or endothelial lining injuries $[1,2]$.

Hypercoagulable states including thrombophilia, malignancy and rheumatologic diseases were considered to be important independent risk factors for the development of CVT [6]. The coincidence of acquired risk factors and inherited thrombophilia was reported to multiply the risk of developing CVT $[2,8]$. D-dimer concentrations may be raised in most patients with CVT, but normal D-dimer levels do not exclude CVT in patients with high suspicion or in the subacute or chronic phase of the disease because D-dimer levels decline with time from onset of symptoms [9]. Elevated plasma level of Factor VIII activity was known to increase the risk of de novo and recurrent venous thromboembolism [10]. It was also previously reported that elevated plasma Factor VIII was the most common prothrombotic risk factor for CVT [11]. In this case, persistently elevated plasma Factor VIII was an important identifiable risk factor for posttraumatic non-pyogenic CVT. The cause of elevated Factor VIII was supposed to be a combination of hereditary and acquired variables. The mechanisms underlying the link between elevated plasma Factor VIII activity and CVT remain to be elucidated.

The patient in this case was successfully treated with endovascular mechanical thrombectomy followed by anti-coagulation involving intravenous UFH and oral warfarin. Endovascular mechanical thrombectomy with balloon angioplasty was reported to resolve post-traumatic CVT and make successful immediate reduction of intracranial pressure [12]. However, there has been insufficient evidence to support the use of endovascular mechanical thrombectomy in patients with CVT. Both American Stroke Association guideline and European Stroke Organization guideline recommend that endovascular mechanical thrombectomy for the management of CVT should be considered only if patients clinically deteriorate despite adequate anti-coagulation or if mass effect made by cerebral venous infarction or ICH causes intracranial hypertension resistant to medical therapies $[13,14]$. Efficacy and safety of endovascular mechanical thrombectomy remain to be elucidated in prospective randomized clinical trial. It should be also addressed whether Nonvitamin $\mathrm{K}$ antagonist Oral Anti-Coagulant (NOAC) could be safely applied to patients with CVT [15].

\section{Conclusion}

Because clinical presentations of CVT are highly variable, the diagnosis of CVT is often delayed or overlooked. We should consider that patients with recent head trauma history without skull fracture and coexistent hypercoagulability could develop CVT that might bring about a fatal complication of cerebral venous infarction with hemorrhagic transformation even when the patient showed no definite focal neurologic deficit or the patient's initial CT scan revealed no intracranial hemorrhage. The novel point in this paper is to suggest the possible pathophysiology underlying CVT after head trauma without skull fracture. The hypercoagulable state triggered by head trauma without skull fracture in patients with pre-existent hypercoagulable conditions like elevated plasma level of Factor VIII activity could synergistically result in devastating CVT. We propose to check the coagulopathy panel including plasma Factor VIII activity in addition to routine lab including PT, aPTT and D-dimer in ER to evaluate the underlying hypercoagulability and the possibility of CVT in patients with recent head trauma history. 


\section{References}

1. Carrie AW, Jaffe FA. Thrombosis of superior sagittal sinus caused by trauma without penetrating injury. J Neurosurg. 1954; 11: 173-182.

2. Rich C, Gill JC, Wernick S, Konkol RJ. An unusual cause of cerebral venous thrombosis in a four-year-old child. Stroke. 1993; 24: 603-605.

3. Delgado Almandoz JE, Kelly HR, Schaefer PW, Lev MH, Gonzalez RG, Romero JM. Prevalence of traumatic dural venous sinus thrombosis in high-risk acute blunt head trauma patients evaluated with multidetector ct venography. Radiology. 2010; 255: 570-577.

4. Hsu PJ, Lee CW, Tang SC, Jeng JS. Pearls \& oy-sters: Delayed traumatic intracerebral hemorrhage caused by cerebral venous sinus thrombosis. Neurology. 2014; 83: e135-137.

5. Maegele M, Schochl H, Menovsky T, Maréchal H, Marklund N, Buki A, et al. Coagulopathy and haemorrhagic progression in traumatic brain injury: Advances in mechanisms, diagnosis, and management. Lancet Neurol. 2017; 16: 630-647.

6. Saadatnia M, Fatehi F, Basiri K, Mousavi SA, Mehr GK. Cerebral venous sinus thrombosis risk factors. Int J Stroke. 2009; 4: 111-123.

7. Ochagavia AR, Boque MC, Torre C, Alonso S, Sirvent JJ. Dural venous sinus thrombosis due to cranial trauma. Lancet. 1996; 347: 1564.

8. Schutta HS, Williams EC, Baranski BG, Sutula TP. Cerebral venous thrombosis with plasminogen deficiency. Stroke. 1991; 22: 401-405.
9. Bushnell C, Saposnik G. Evaluation and management of cerebral venous thrombosis. Continuum (Minneap Minn). 2014; 20: 335-351.

10. Kyrle PA, Minar E, Hirschl M, Bialonczyk C, Stain M, Schneider B, et al. High plasma levels of factor viii and the risk of recurrent venous thromboembolism. N Engl J Med. 2000; 343: 457-462.

11. Bugnicourt JM, Roussel B, Tramier B, Lamy C, Godefroy O. Cerebral venous thrombosis and plasma concentrations of factor viii and von willebrand factor: A case control study. J Neurol Neurosurg Psychiatry. 2007; 78: 699-701.

12. Bishop FS, Finn MA, Samuelson M, Schmidt RH. Endovascular balloon angioplasty for treatment of posttraumatic venous sinus thrombosis. Case report. J Neurosurg. 2009; 111: 17-21.

13. Saposnik G, Barinagarrementeria F, Brown RD Jr, Bushnell CD, Cucchiara $B$, Cushman $M$, et al. Diagnosis and management of cerebral venous thrombosis: A statement for healthcare professionals from the american heart association/american stroke association. Stroke. 2011; 42: 1158-1192.

14. Ferro JM, Bousser MG, Canhao P, Coutinho JM, Crassard I, Dentali F, et al. European stroke organization guideline for the diagnosis and treatment of cerebral venous thrombosis-endorsed by the European academy of neurology. Eur J Neurol. 2017; 24: 1203-1213.

15. Silvis SM, de Sousa DA, Ferro JM, Coutinho JM. Cerebral venous thrombosis. Nat Rev Neurol. 2017; 13: 555-565.
Austin J Cerebrovasc Dis \& Stroke - Volume 6 Issue 1 - 2019 ISSN : 2381-9103 | www.austinpublishing group.com

Koo et al. (C) All rights are reserved
Citation: Jeon K, Kim JH, Lee K, Park K-N, Kim B-S, Shin YS, et al. Elevated Plasma Factor VIII in Non-Pyogenic Cerebral Venous Thrombosis after Head Trauma without Skull Fracture. Austin J Cerebrovasc Dis \& Stroke. 2019; 6(1): 1082 